\title{
Approval Voting and Scoring Rules with Common Values*
}

\author{
David S. Ahn ${ }^{\dagger}$ \\ Santiago Oliveros ${ }^{\ddagger}$ \\ University of California, Berkeley \\ University of Essex
}

August 2016

\begin{abstract}
We compare approval voting with other scoring rules for environments with common values and private information. For finite electorates, the best equilibrium under approval voting is superior to plurality rule or negative voting. For large electorates, if any scoring rule yields a sequence of equilibria that efficiently aggregates information, then approval voting must do so as well.
\end{abstract}

\section{Introduction}

We compare the ability of approval voting to aggregate private information in elections with common values versus other scoring rules such as plurality rule or the Borda count. Our main results demonstrate the advantages of approval voting in aggregating information and their proofs illuminate a basic mechanism: its flexibility allows approval voting to outperform plurality or negative voting in finite elections, and in large elections, mixing ballots under approval voting can approximate the outcome of an arbitrary scoring rule.

Our paper adds to an active literature that studies information aggregation for multiple candidates, where a common theme is the superiority of approval voting over other institutions. ${ }^{1}$ This comparison is currently understood mainly for special environments with specific restrictions on the support of possible values. Goertz and Maniquet (2011) consider a class of environments where voters are indifferent between the two inferior candidates. Within this class, approval voting is the only scoring rule that admits an informationally efficient limit equilibrium. Bouton and Castanheira (2012) consider the divided majority problem, where a majority block of voters shares a

\footnotetext{
*We acknowledge the National Science Foundation for financial support under Grant SES-0851704.

${ }^{\dagger}$ Department of Economics, University of California, 530 Evans Hall \#3880, Berkeley, CA 94720-3880. Email: dahn@econ. berkeley.edu.

${ }^{\ddagger}$ Department of Economics, 5B. 118, University of Essex, Wivenhoe Park, Colchester, CO4 3SQ. Email: soliveb@essex.ac.uk.

${ }^{1} \mathrm{~A}$ related literature considers approval voting as a method to aggregate preferences with private values. For example, Giles and Postl (2014) characterize Bayesian equilibria and evaluate approval voting within the class of all scoring rules.
} 
common preference for two candidates over a third minority candidate but has incomplete information about which of the two preferred candidates is superior, and show that approval voting yields a unique limit equilibrium that efficiently aggregates information while plurality rule can have multiple equilibria. This advantage for divided majority problems is shown for small electorates in theory and in experiments by Bouton, Castanheira, and Llorente-Saguer (2016). Our main substantive contribution is to understand the performance of approval voting under arbitrary forms of common preference.

Our main methodological contribution is to adapt the following insight due to McLennan (1998): in a common-value election, a strategy that maximizes utility is an equilibrium. Therefore, any voting rule that provides more flexibility for voters to express their information cannot leave voters any worse off. Approval voting can replicate any plurality rule outcome by having voters submit ballots supporting a singleton set of candidates, so approval voting must be weakly better under common values. For arbitrary scoring rules beyond plurality rule, approval voting can arbitrarily approximate any outcome with appropriate mixing of ballots. The main benefit of our approach is that it illuminates an essential advantage of approval voting in common-value environments, namely the flexibility it affords the voters in adapting their votes to their information.

McLennan's observation makes no assumptions on the information or preferences of the voters, so is general enough to apply to arbitrary environments with common values. However, we do not explicitly construct equilibria and instead focus attention on efficiency bounds. While our assumptions are more general than those in the literature, our conclusions are commensurately less sharp. We cannot speak to the uniqueness of equilibrium nor to its characterization. An analysis of inefficient equilibria is more delicate and requires direct consideration of the environment. For example, Goertz and Maniquet (2011) present one environment with inferior limit equilibria under approval voting while Bouton and Castanheira (2012) show that the unique limit equilibrium of approval voting for the divided majority problem is efficient.

Our analysis is limited to scoring rules, hence excludes some prominent voting systems such as instant runoff. Simple examples demonstrate that feasible conditional outcomes under instant runoff cannot be replicated by approval voting. ${ }^{2}$ However, these examples are knife-edge when the number of signals is greater than or equal to the number of candidates. For such environments, Barelli, Bhattacharya, and Siga (personal communication, June 16, 2016) recently proved a general efficiency result that establishes as a corollary the efficiency of information aggregation in large elections for a generic set of statistical environments. When there are strictly fewer signals than candidates, efficiency can be mechanically precluded since signals cannot separate the optimal candidate and the comparison of instant runoff to approval voting remains unresolved.

\footnotetext{
${ }^{2}$ We thank an anonymous referee for observing this possibility and providing an example. To our knowledge, he or she is the first to understand this point that had been previously unnoticed in the literature.
} 


\section{Model}

A finite set of $I$ voters must collectively decide a winner among three candidates $K=\{a, b, c\} .{ }^{3}$ Each voter $i$ can submit a ballot, or vote vector, $\left(c_{i}(1), c_{i}(2), c_{i}(3)\right)$, where $c_{i}(k)$ denotes the score allocated by voter $i$ to candidate $k$. A scoring rule is therefore defined by a range of permitted ballots. As in Myerson (2002), we consider $(A, B)$-scoring rules defined by two parameters $0 \leq A \leq$ $B \leq 1$. For a fixed $(A, B)$-scoring rule, each voter can submit either a permutation of $(1, B, 0)$ or of $(1, A, 0)$. Let $C$ denote the space of all possible ballots. Given a profile of ballots $\left(c_{1}, \ldots, c_{I}\right)$, the winner $W\left(c_{1}, \ldots, c_{I}\right)$ the candidates whose total score $\sum_{I} c_{i}(k)$ is maximal. In the case of a tie, uniform randomization is used to select among the winners.

The following $(A, B)$-scoring rules are notable. The case $(A, B)=(0,0)$ is plurality voting, where each voter can support a single candidate but cannot express any intensity of preference between inferior candidates. The case $(A, B)=(1,1)$ is negative voting, where each voter can oppose a single candidate but cannot express preference among superior candidates. The case $(A, B)=(0.5,0.5)$ is the Borda count, where candidates are totally ranked by each voter and receive scores proportional to their ranks. Of particular interest is the case $(A, B)=(0,1)$ which is approval voting, where each voter decides a set of one or two candidates to support.

Let $\Omega$ be a finite set of states of the world. The prior probability of state $\omega \in \Omega$ is $P(\omega)$. All voters share a common utility $U(k \mid \omega)$ for candidate $k$ in state $\omega$. We assume that a unique best candidate $k_{\omega}$ maximizes $U(k \mid \omega)$ for each state $\omega$. Voters do not know the state of the world. The finite set $S$ is a set of possible signals. Given the state $\omega$, each voter receives a conditionally independent signal following the conditional distribution $F(s \mid \omega)$.

A (mixed) strategy $\sigma_{i}: S \rightarrow \Delta C$ assigns a probability over vote vectors to each signal. When $\sigma_{i}(s)$ is a degenerate lottery with probability one of $c$, we slightly abuse notation and write $\sigma_{i}(s)=c$. A profile $\boldsymbol{\sigma}=\left(\sigma_{1}, \ldots, \sigma_{I}\right)$ of strategies is symmetric if $\sigma_{i}=\sigma_{j}$ for all $i, j$. When referring to symmetric strategy profiles, we drop the voter subscript. The common expected utility for the strategy profile $\boldsymbol{\sigma}(\mathbf{s})=\sigma_{1}\left(s_{1}\right), \ldots, \sigma_{I}\left(s_{I}\right)$ is

$$
E U(\boldsymbol{\sigma})=\int_{\Omega} \int_{S} \int_{\mathcal{X}^{I}} U\left(W\left(c_{1}, \ldots, c_{I}\right) \mid \omega\right) d \boldsymbol{\sigma}(\mathbf{s}) d F(\mathbf{s} \mid \omega) d P(\omega) .
$$

A symmetric equilibrium is a symmetric strategy profile where no single voter can strictly improve the common expected utility by changing her strategy. Consider a sequence of symmetric strategy profiles $\left(\sigma_{I}\right)$ indexed by population size $I$. We say that the probability of error goes to zero if, for every $\omega$,

$$
\int_{S} \int_{\mathcal{X}^{I}} U\left(W\left(c_{1}, \ldots, c_{I}\right)|\omega| \omega\right) d \boldsymbol{\sigma}_{I}\left(\mathbf{s}_{I}\right) d F\left(\mathbf{s}_{I} \mid \omega\right) \longrightarrow U\left(k_{\omega} \mid \omega\right)
$$

as $I$ goes to infinity. The probability of error goes to zero if and only if the probability of the best candidate $k_{\omega}$ winning the election goes to one for every state of the world.

\footnotetext{
${ }^{3}$ We assume three candidates for ease of exposition. Suitable versions of our results hold for an arbitrary number of candidates.
} 


\section{Results}

Our first result asserts that the maximal equilibrium utility under approval voting is greater than or equal to the maximal equilibrium utility under plurality rule or under negative voting.

Proposition 1. If $\sigma^{*}$ is a symmetric equilibrium for plurality rule or for negative voting, then there exists a symmetric equilibrium $\rho^{*}$ of approval voting such that $E U\left(\rho^{*}\right) \geq E U\left(\sigma^{*}\right)$.

Proof. Suppose $\sigma^{*}$ is a symmetric equilibrium of plurality rule. Define the strategy profile $\rho$ for approval voting by $\rho(s)=\sigma^{*}(s){ }^{4}$ The expected utility of $\rho$ is identical to the expected utility of $\sigma^{*}$. Since $U$ is continuous and the space of symmetric strategies is compact, there exists a symmetric strategy profile $\rho^{*}$ that maximizes the common expected utility $U$ for approval voting among all symmetric strategy profiles. By Theorem 2 of McLennan (1998), $\rho^{*}$ is an equilibrium for approval voting. By construction, $E U\left(\rho^{*}\right) \geq E U(\rho)=E U\left(\sigma^{*}\right)$.

The argument for negative voting is identical.

While the reasoning for Proposition 1 is mathematically uncomplicated, to our knowledge it has not been previously observed. Specifically, any ballot that can be submitted under plurality rule can also be submitted under approval voting. So the expected utility under plurality rule can be replicated under approval voting, by having each voter approve the singleton set corresponding to the candidate that she would support under plurality rule. Of course, approval voting also allows ballots supporting two candidates. However, McLennan (1998) observed that the best equilibrium in a game of common values must be as good as any strategy profile. So the best equilibrium under approval voting must be at least as efficient as any equilibrium under plurality rule. The argument makes transparent the connection between approval voting and plurality rule: the fundamental reason that approval voting outperforms plurality rule (or negative voting) is that approval voting allows a larger set of feasible ballots. While similar arguments for approval voting are suggested in private value environments, its flexibility has direct force in common-value environments where the large set of possible ballots allows voters to replicate any outcome under plurality rule.

Our second finding is that the best asymptotic equilibrium under approval voting is at least as good as the best asymptotic equilibrium under any interior $(A, B)$-scoring rule where $0<A \leq B<$ 1. Unlike plurality rule or negative voting, the strategies under general $(A, B)$-scoring rules cannot be exactly replicated by approval voting. When $A \neq B$, a general $(A, B)$-scoring rule allows for twelve distinct vote vectors, while approval voting allows only for six. However, the outcome of the $(A, B)$-scoring rule can be approximated through appropriate randomization of the ballots under approval voting. Specifically, the expected vote counts can be maintained under approval voting. This approximation becomes asymptotically precise, so the limit outcome under a general scoring rule can be replicated by approval voting.

\footnotetext{
${ }^{4}$ To be pedantic, we identify $\sigma^{*}(s)$ within the strictly large strategy simplex for approval voting.
} 
Proposition 2. Suppose $A, B \in(0,1)$. If there exists a sequence $\left(\sigma_{I}\right)$ of symmetric strategies for the $(A, B)$ scoring rule that takes the error probability to zero, then there exists a sequence $\left(\rho_{I}^{*}\right)$ of symmetric equilibria for approval voting that takes the error probability to zero. ${ }^{5}$

Proof. For any symmetric strategy profile $\sigma$ of the $(A, B)$ scoring rule, define the symmetric strategy profile $\rho$ of approval voting with permutations of the following:

$$
\begin{aligned}
& {[\rho(s)](1,1,0)=\sum_{X=A, B} X\{[\sigma(s)](1, X, 0)+[\sigma(s)](X, 1,0)\}} \\
& {[\rho(s)](1,0,0)=\sum_{X=A, B}(1-X)\{[\sigma(s)](1, X, 0)+[\sigma(s)](1,0, X)\}}
\end{aligned}
$$

Without loss of generality, fix a state $\omega$ such that $k_{\omega}=a$. We now prove that the limit probability that candidate $a$ wins the election goes to one, conditional on the state $\omega$. All probabilities and expectations hereon are conditional on $\omega$. Define the random variable

$$
\delta_{I i}=\sigma_{I i} \cdot(1,0,0)-\sigma_{I i} \cdot(0,1,0)
$$

where the "." operation denotes the dot product. The random variable $\delta_{I i}$ is the difference in the scores given to candidate $a$ and candidate $b$ by voter $i$ when playing the strategy $\sigma_{I}$ in the $(A, B)$-scoring rule. Similarly, let

$$
\Delta_{I i}=\rho_{I i} \cdot(1,0,0)-\rho_{I i} \cdot(0,1,0)
$$

i.e. the difference in the scores of candidate $a$ and candidate $b$ when playing the strategy $\rho_{I}$ under approval voting.

We first show that the conditional expectations of the score differences are identical under $\sigma$ and $\rho$.

Lemma 1. $\mathbf{E}\left(\delta_{I i}\right)=\mathbf{E}\left(\Delta_{I i}\right)$.

Proof. We will show that the expectations conditional on a fixed signal are equal: $\mathbf{E}\left(\delta_{I i} \mid s_{i}=s\right)=$ $\mathbf{E}\left(\Delta_{I i} \mid s_{i}=s\right)$ for all signals $s_{i}$. Then the unconditional expectations are also equal.

First, computing the expectation for the $(A, B)$-scoring rule:

$$
\begin{aligned}
\mathbf{E}\left(\delta_{I i} \mid s_{i}=s\right)= & \sum_{X=A, B}\left\{\left[\sigma_{I}(s)\right](1,0, X)-\left[\sigma_{I}(s)\right](0,1, X)\right\} \\
& +\sum_{X=A, B} X\left\{\left[\sigma_{I}(s)\right](X, 0,1)-\left[\sigma_{I}(s)\right](0, X, 1)\right\} \\
& +\sum_{X=A, B}(1-X)\left\{\left[\sigma_{I}(s)\right](1, X, 0)-\left[\sigma_{I}(s)\right](X, 1,0)\right\}
\end{aligned}
$$

\footnotetext{
${ }^{5}$ The interiority of the scoring rule is required only to ensure that strategies provide some but not equal support for the middle candidate. As the proof should make clear, approval voting can approximate the outcome of any interior voting strategy; the interiority of allowed scores mechanically ensures this case.
} 
Next, using the construction of $\rho_{I}$ in (1) and (2) for approval voting:

$$
\begin{aligned}
\mathbf{E}\left(\Delta_{I i} \mid s_{i}=s\right)= & {\left.\left[\rho_{I}(s)\right](1,0,0)+\left[\rho_{I}(s)\right](1,0,1)-\left[\rho_{I}(s)\right](0,1,0)-\left[\rho_{I}(s)\right](0,1,1)\right] } \\
= & \sum_{X=A, B}(1-X)\left\{\left[\sigma_{I}(s)\right](1, X, 0)+\left[\sigma_{I}(s)\right](1,0, X)\right\} \\
& +\sum_{X=A, B} X\left\{\left[\sigma_{I}(s)\right](1,0, X)+\left[\sigma_{I}(s)\right](X, 0,1)\right\} \\
& -\sum_{X=A, B}(1-X)\left\{\left[\sigma_{I}(s)\right](X, 1,0)+\left[\sigma_{I}(s)\right](0,1, X)\right\} \\
& -\sum_{X=A, B} X\left\{\left[\sigma_{I}(s)\right](0,1, X)+\left[\sigma_{I}(s)\right](0, X, 1)\right\}
\end{aligned}
$$

Basic algebra confirms that the right hand sides are equal to each other.

Let $\mathbf{V}(\cdot)$ denote the variance of a random variable, again conditional on $\omega$. Consider the case where $\lim \mathbf{V}\left(\delta_{I i}\right) \rightarrow 0$ for sufficiently large $I$. Then $\delta_{I i}$ converges to a point mass on some constant $E$ for large $I$. However, because $(A, B)$ are interior, 0 is not part of the support of $\delta_{I i}$ because it is impossible for a ballot to provide equal scores to candidates $a$ and $b$. Therefore, since the probability that $\sum_{I i} \delta_{I i}>0$ goes to one, it must be the case that the point mass is suppored at a strictly positive point. This point defines the expectation $\lim \mathbf{E}\left(\delta_{I i}\right)=E>0$ for large enough $I$. By Lemma 1, it is also the case that $\lim \mathbf{E}\left(\Delta_{I i}\right)=E>0$ for large enough $I$. By the weak law of large numbers for triangular arrays, the probability that $\sum_{i=1}^{I} \Delta_{I i} / I>0$ goes to one. Hence the probability $\sum_{i=1}^{I} \Delta_{I i}>0$ also goes to one.

So, without loss of generality, suppose $\lim \mathbf{V}\left(\delta_{I i}\right)>0$ for all $I, i$. First, note that:

$$
\operatorname{Pr}\left(\sum_{i=1}^{I} \delta_{I i} \leq 0\right)=\operatorname{Pr}\left(\frac{\sum_{i=1}^{I} \delta_{I i}-I \mathbf{E}\left(\delta_{I i}\right)}{\sqrt{I \mathbf{V}\left(\delta_{I i}\right)}} \leq-\frac{I \mathbf{E}\left(\delta_{I i}\right)}{\sqrt{I \mathbf{V}\left(\delta_{I i}\right)}}\right)
$$

By the Central Limit Theorem for triangular arrays (Billingsley 1995, Theorem 27.2):

$$
\left|\operatorname{Pr}\left(\frac{\sum_{i=1}^{I} \delta_{I i}-I \mathbf{E}\left(\delta_{I i}\right)}{\sqrt{I \mathbf{V}\left(\delta_{I i}\right)}} \leq-\frac{I \mathbf{E}\left(\delta_{I i}\right)}{\sqrt{I \mathbf{V}\left(\delta_{I i}\right)}}\right)-\Phi\left(-\frac{I \mathbf{E}\left(\delta_{I i}\right)}{\sqrt{I \mathbf{V}\left(\delta_{I i}\right)}}\right)\right| \rightarrow 0
$$

Since $\operatorname{Pr}\left(\sum_{i=1}^{I} \delta_{I i} \leq 0\right)$ must go to zero by the assumed efficiency of $\sigma_{I}$, the triangle inequality implies that $\Phi\left(-\frac{I \mathbf{E}\left(\delta_{I i}\right)}{\sqrt{I \mathbf{V}\left(\delta_{I i}\right)}}\right)$ must go to zero. Hence:

$$
\sqrt{I} \frac{\mathbf{E}\left(\delta_{I i}\right)}{\sqrt{\mathbf{V}\left(\delta_{I i}\right)}} \rightarrow \infty
$$


Then

$$
\begin{aligned}
\sqrt{I} \frac{\mathbf{E}\left(\Delta_{I i}\right)}{\sqrt{\mathbf{V}\left(\Delta_{I i}\right)}} & =\sqrt{I} \frac{\mathbf{E}\left(\delta_{I i}\right)}{\sqrt{\mathbf{V}\left(\Delta_{I i}\right)}}, \text { by Lemma } 1 \\
& =\sqrt{I} \frac{\mathbf{E}\left(\delta_{I i}\right)}{\sqrt{\mathbf{V}\left(\delta_{I i}\right)}} \times \sqrt{\frac{\mathbf{V}\left(\delta_{I i}\right)}{\mathbf{V}\left(\Delta_{I i}\right)}}
\end{aligned}
$$

The second factor $\sqrt{\frac{\mathbf{V}\left(\delta_{I i}\right)}{\mathbf{V}\left(\Delta_{I i}\right)}}$ is strictly positive. It is uniformly bounded away from zero, since $\lim \mathbf{V}\left(\delta_{I i}\right)>0$ and $\mathbf{V}\left(\Delta_{I i}\right)$ is uniformly bounded $\Delta_{I i}$ takes values in a bounded set $[-1,1]$. Since the first factor $\frac{\mathbf{E}\left(\delta_{I i}\right)}{\sqrt{\mathbf{V}\left(\delta_{I i}\right)}}$ goes to infinity, so does the product:

$$
\sqrt{I} \frac{\mathbf{E}\left(\Delta_{I i}\right)}{\sqrt{\mathbf{V}\left(\Delta_{I i}\right)}} \rightarrow \infty
$$

Hence $\Phi\left(-\sqrt{I} \frac{\mathbf{E}\left(\Delta_{I i}\right)}{\sqrt{\mathbf{V}\left(\Delta_{I i}\right)}}\right) \rightarrow 0$. Applying the Central Limit Theorem for triangular arrays and the triangle inequality as before, we have that $\operatorname{Pr}\left(\sum_{i=1}^{I} \Delta_{I i} \leq 0\right)$ goes to zero.

So the probability of the complementary event $\sum_{i=1}^{I} \Delta_{I i}>0$, where the total score for candidate $a$ is strictly larger than the total score for candidate $b$, must go to one. Similarly, the probability that the total score for candidate $a$ is strictly larger than the total score for candidate $c$ also goes to one. The intersection event is that candidate $a$ wins the election, and the probability of this event goes to one.

By Theorem 2 of McLennan (1998), there must exist a sequence of symmetric equilibrium $\rho_{I i}^{*}$ that provides weakly more common utility than $\rho_{I i}$ for each fixed population $I$. But since $\rho_{I i}$ takes the probability of error to zero, then $\rho_{I i}^{*}$ must also take the probability of error to zero.

\section{References}

Billingsley, P. (1995): Probability and Measure. Wiley, New York.

Bouton, L., And M. Castanheira (2012): "One Person, Many Votes: Divided Majority and Information Aggregation," Econometrica, 80, 43-87.

Bouton, L., M. Castanheira, And A. Llorente-Saguer (2016): "Divided Majority and Information Aggregation: Theory and Experiment," Journal of Public Economics, 134, 114-128.

Giles, A., AND P. Postl (2014): "Equilibrium and Effectiveness of Two-Parameter Scoring Rules," Mathematical Social Sciences, 68, 31-52.

Goertz, J. M., And F. Maniquet (2011): "On the Informational Efficiency of Simple Scoring Rules," Journal of Economic Theory, 146, 1464-1480.

McLennan, A. (1998): "Consequences of the Condorcet Jury Theorem for Beneficial Information Aggregation by Rational Agents," American Political Science Review, 92, 413-418.

Myerson, R. B. (2002): "Comparison of Scoring Rules in Poisson Voting Games," Journal of Economic Theory, $103,219-251$. 